\title{
Donor sex, age and ethnicity impact stored red blood cell antioxidant metabolism through mechanisms in part explained by glucose 6-phosphate dehydrogenase levels and activity
}

Haematologica 2021

Volume 106(5):1290-1302

\section{Correspondence:}

ANGELO D'ALESSANDRO

angelo.dalessandro@ucdenver.edu

Received: January 7, 2020.

Accepted: March 27, 2020.

Pre-published: April 2, 2020.

https://doi.org/10.3324/haematol.2020.246603

(C)2021 Ferrata Storti Foundation

Material published in Haematologica is covered by copyright. All rights are reserved to the Ferrata Storti Foundation. Use of published material is allowed under the following terms and conditions:

https://creativecommons.org/licenses/by-nc/4.0/legalcode. Copies of published material are allowed for personal or internal use. Sharing published material for non-commercial purposes is subject to the following conditions:

https://creativecommons.org/licenses/by-nc/4.0/legalcode, sect. 3. Reproducing and sharing published material for commercial purposes is not allowed without permission in writing from the publisher.

\author{
Angelo D'Alessandro, ${ }^{1,2,3}$ Xiaoyun Fu, ${ }^{4}$ Tamir Kanias ${ }^{3,5}$ Julie A. Reisz, ${ }^{1}$ \\ Rachel Culp-Hill, ${ }^{1}$ Yuelong Guo, ${ }^{6}$ Mark T. Gladwin, ${ }^{5}$ Grier Page, ${ }^{6}$ \\ Steve Kleinman, ${ }^{7}$ Marion Lanteri, ${ }^{8}$ Mars Stone, ${ }^{8}$ Michael P. Busch, ${ }^{8}$ \\ and James C. Zimring ${ }^{9 \#}$ for the Recipient Epidemiology and Donor Evaluation \\ Study-III (REDS III)
}

\begin{abstract}
${ }^{1}$ Department of Biochemistry and Molecular Genetics, University of Colorado Denver Anschutz Medical Campus, Aurora, CO, USA; ${ }^{2}$ Department of Medicine - Division of Hematology, University of Colorado Denver - Anschutz Medical Campus, Aurora, CO, USA; ${ }^{3}$ Vitalant Research Institute (previously Blood Systems Research Institute), Denver, CO, USA; 'Bloodworks Northwest Research Institute, Seattle, WA, USA; ${ }^{4}$ University of Pittsburgh, Pittsburgh, PA, USA; ${ }^{6}$ RTI International, Atlanta, GA, USA; 'University of British Columbia, Victoria, Canada; ${ }^{8}$ Vitalant Research Institute (previously Blood Systems Research Institute), San Francisco, CA, USA and 'University of Virginia, Charlottesville, VA, USA

\#MPB and JCZ contributed equally as co-senior authors
\end{abstract}

\section{ABSTRACT}

$\mathrm{R}$ ed blood cell (RBC) storage in the blood bank promotes the progressive accumulation of metabolic alterations that may ultimately impact the erythrocyte capacity to cope with oxidant stressors. However, the metabolic underpinnings of the capacity of $\mathrm{RBC}$ to resist oxidant stress and the potential impact of donor biology on this phenotype are not known. Within the framework of the REDS-III RBC-Omics study, RBC from 8,502 healthy blood donors were stored for 42 days and tested for their propensity to hemolyse following oxidant stress. A subset of extreme hemolysers donated a second unit of blood, which was stored for 10,23, and 42 days and profiled again for oxidative hemolysis and metabolomics (599 samples). Alterations of RBC energy and redox homeostasis were noted in donors with high oxidative hemolysis. RBC from females, donors over 60 years old, donors of Asian/South Asian race-ethnicity, and RBC stored in additive solution-3 were each independently characterized by improved antioxidant metabolism compared to, respectively, males, donors under 30 years old, Hispanic and African American race ethnicity donors, and RBC stored in additive solution-1. Merging metabolomics data with results from an independent genome-wide association study on the same cohort, we identified metabolic markers of hemolysis and glucose 6-phosphate dehydrogenasedeficiency, which were associated with extremes in oxidative hemolysis and dysregulation in nicotinamide adenine dinucleotide phosphate and glutathione-dependent detoxification pathways of oxidized lipids. Donor sex, age, ethnicity, additive solution and glucose 6-phosphate dehydrogenase status impact the metabolism of the stored erythrocyte and its susceptibility to hemolysis following oxidative insults.

\section{Introduction}

Over the past decade the application of Omics Technologies ${ }^{1}$ to the field of red blood cell (RBC) storage has exponentially expanded our understanding of the temporal sequence and mechanisms of the storage lesion. Indeed, while refrigerated storage in the blood bank is a logistic necessity to make 110 million units 
available for transfusion every year worldwide, the process comes at a significant cost in terms of RBC structural $^{2-4}$ and biochemical homeostasis. ${ }^{1}$ Some of these "storage lesions" are inevitable, since refrigeration temperatures negatively impact the activity of key enzymes regulating red cell energy and ion pump homeostasis. ${ }^{5}$ In small scale studies, the metabolic lesion has been reproducibly assessed to a quantifiable extent, which allowed the definition of metabolic markers of the so-called "metabolic age" of stored RBC. ${ }^{2,6}$ Despite the consistency of these laboratory observations, it is still a matter of debate whether the (metabolic) storage lesion could represent an etiological contributor to (or a reliable predictor of) transfusion outcomes in the recipient. ${ }^{7}$ Indeed, storage-induced impairments in the homeostasis of high energy phosphate compounds adenosine triphosphate (ATP) and 2,3-diphosphoglycerate (DPG) should negatively impact RBC capacity to bind and off-load oxygen upon transfusion. ${ }^{8}$ Depletion of ATP and DPG could represent a concern in massively transfused patients, since the rate at which these compounds are replenished within the first 72 hours (h) upon transfusion may not be sufficient to meet the oxygen metabolic demands in severely hypoxic recipients. ${ }^{9,10}$ Studies in animal models and humans have shown that some small molecule metabolites could represent reliable correlates to Food and Drug Administration gold standards for stored blood quality, i.e., storage hemolysis ${ }^{11,12}$ and post-transfusion recovery. ${ }^{13,14}$ For example, metabolites like hypoxanthine, an ATP-breakdown and oxidation product, have been correlated to hemolysis and post-transfusion recoveries in mice and humans. ${ }^{13}$ Similar correlations have been reported for lipid oxidation products. ${ }^{14}$

Despite the overwhelming evidence from in vitro studies, randomized clinical trials ${ }^{15}$ have hitherto failed to capture any signal associated with poorer outcomes when comparing transfusion of the freshest available units versus the standard of practice. On the other hand, a recent analysis of a linked donor and recipient database indicated that transfusion of RBC units less than 35 days old was associated with a higher recipient hemoglobin increment compared to transfusion of 35- to 42-day old $\mathrm{RBC}$ units. ${ }^{16}$ The apparent inconsistencies among the studies on the age of blood in the literature could be reconciled by the appreciation of the fact that RBC, like people, do not always age the same. ${ }^{17}$ In other terms, the molecular age of blood may be a distinct parameter from the storage age calculated in days since the time of donation. ${ }^{18}$ Biological variability in donors ${ }^{19}$ and different $\mathrm{RBC}$ component processing strategies ${ }^{20}$ may for example impact hemoglobin oxygen saturation across donors/components, ${ }^{21}$ which in turn affects RBC susceptibility to oxidative stress during storage. ${ }^{13,22,23}$ Small-scale laboratory studies corroborated the hypothesis that RBC antioxidant capacity ${ }^{24}$ and storage-induced susceptibility to oxidative stress may indeed be donor-dependent. ${ }^{25}$ This statement holds true when considering some categories of routinely accepted donors who are more susceptible to storage-induced oxidative stress owing to common enzymopathies. For example, deficiency of glucose 6-phosphate dehydrogenase (G6PD) activity affects $\sim 400$ million people worldwide, including $\sim 10 \%$ of the African American donor population in some metropolitan areas. $^{26}$ These subjects are characterized by a decreased capacity to activate the pentose phosphate pathway (PPP) and thus to generate the nicotinamide adenine dinucleotide phosphate (NADPH) necessary to reduce oxidized glutathione (GSH) and NADPH-dependent antioxidant enzymes. ${ }^{26}$ RBC from G6PD deficient donors are characterized by altered energy and redox metabolism, ${ }^{27,28}$ a feature that has been preliminarily associated with poorer capacity to circulate upon transfusion to sickle cell recipients ${ }^{29}$ and poorer post-transfusion recoveries in autologous volunteers. ${ }^{30}$ As such, population screening in regions where the prevalence of G6PD deficiency is $3-5 \%$ or greater (in males) is recommend by the World Health Organization, ${ }^{31}$ but no specific screening for G6PD activity is routinely in place for blood donors in the United States.

In the past few years, large scale studies have been designed to focus on the impact of donor biology on storage quality and transfusion outcomes. Within the framework of the National Heart Lung and Blood Institute (NHLBI, NIH) Recipient Epidemiology and Donor Evaluation Study (REDS)-III RBC-Omics study, four blood centers across the United States enrolled $\sim 13,800$ healthy donor volunteers of different ages, sex and ethnicities. Preliminary analyses of the data obtained from this cohort allowed us to conclude that (i) donor sex (and testosterone levels ${ }^{32}$ ), age and ethnicity impact the hemolytic propensity of stored RBC; $;^{33,34}$ (ii) stored RBC from multiple units donated by the same donors have a similar propensity to hemolyze following pro-oxidant or osmotic insults; ${ }^{35}$ and (iii) the storage duration contributes to explain $\sim 13 \%$ of the total metabolic heterogeneity of stored $\mathrm{RBC}$, a percentage similar to the impact noted for storage additives in a subgroup of recalled donors from the original RBC-Omics cohort. ${ }^{36}$

In the light of this background, the continued characterization of the impact of donor biology on storability and transfusion outcomes is a critical step towards the establishment of personalized transfusion medicine practices.

\section{Methods}

\section{REDS-III RBC-Omics study participants and samples}

Donor selection and recruitment for the RBC-Omics study under approved protocols (BioLINCC Study: HLB02071919a) were previously detailed. ${ }^{35,37,38}$ Donors were enrolled at the four participating REDS-III US blood centers. Overall, 13,758 whole blood donors were enrolled and 13,403 (97\%) age 18+ provided informed consent to participate in the study; of these, 8,502 were evaluated for oxidative hemolysis on RBC stored for $\sim 39$. 42 days. Extreme hemolysers ( $5^{\text {th }}$ and $95^{\text {th }}$ percentile) from the donors tested for end of storage oxidative hemolysis were asked to donate a second unit of blood. These units were sterilely sampled at storage day 10, 23 and 42 for oxidative hemolysis and metabolomics analyses (599 samples in total). Blood collection, sample processing and other aspects of the screening and recall phases of the RBC-Omics study have been extensively described. . $^{33,34}$

\section{Oxidative hemolysis}

Oxidative hemolysis was determined at the University of Pittsburgh and Vitalant Research Institute as reported and further detailed in references. Briefly, RBC were incubated with 2,2'-azobis-2-methyl-propanimidamide, dihydrochloride (AAPH, $150 \mathrm{mmoL}$ ) to determine susceptibility to oxidative hemolysis, as extensively described. ${ }^{33}$ 


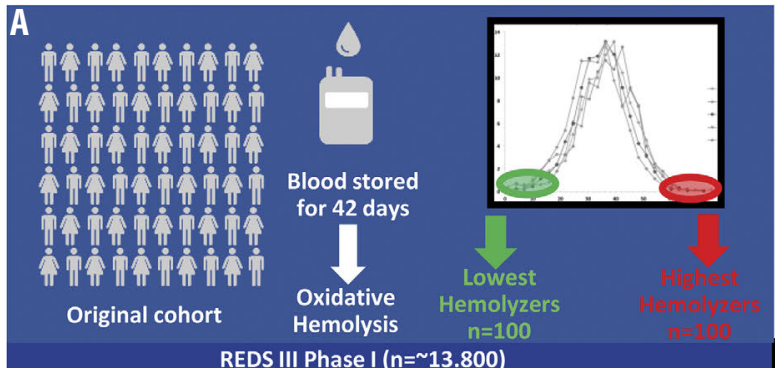

B

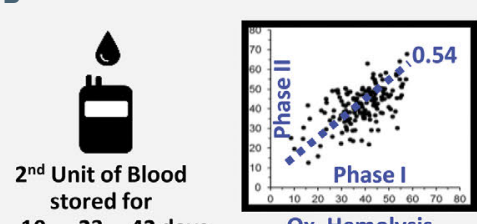
$10 \quad 2342$ days Recalled donors

Ox. Hemolysis

Reproducbility

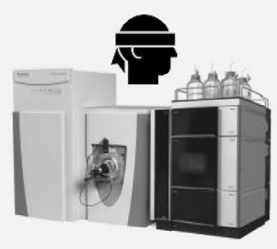

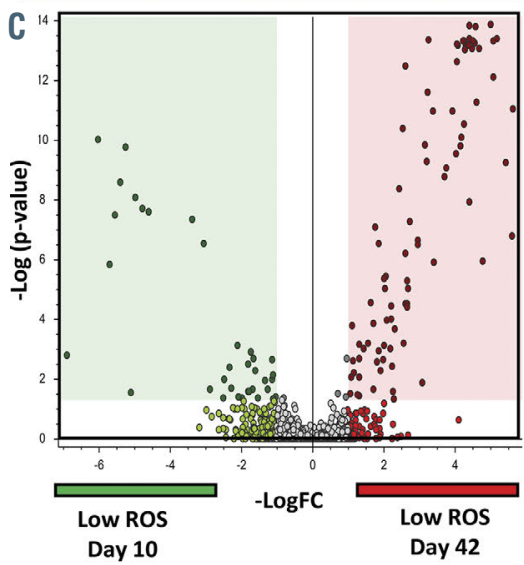

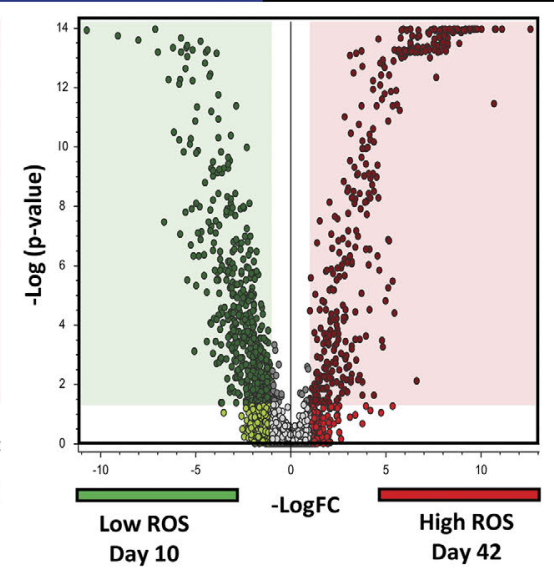

REDS III Phase II ( $\mathrm{n}=599)$

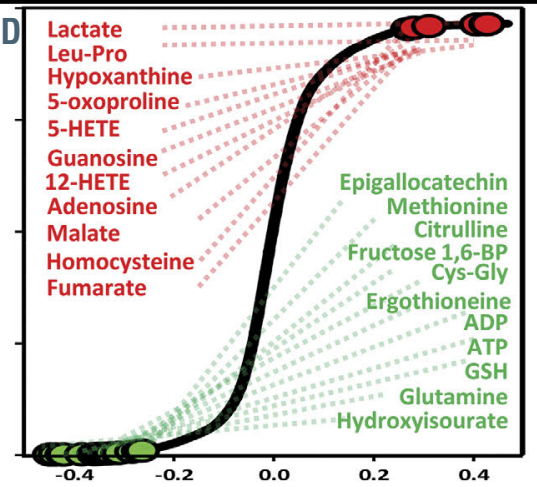

Correlation to Oxidative Hemolysis

E RBC stored in ADDITIVE SOLUTION 1 ( $n=154$ )

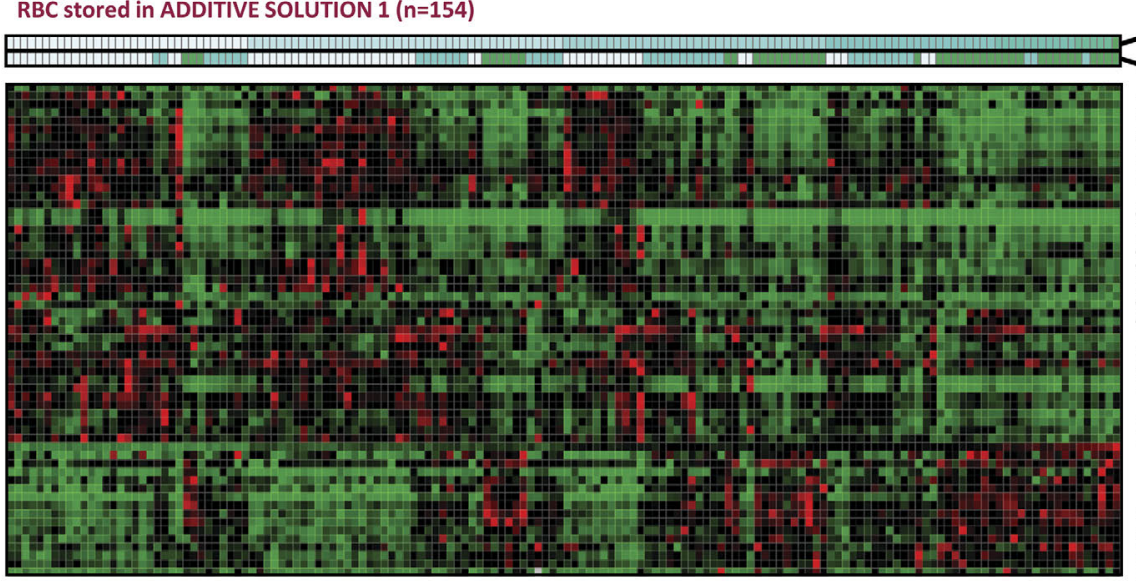

F RBC stored in ADDITIVE SOLUTION 3 ( $n=455$ )
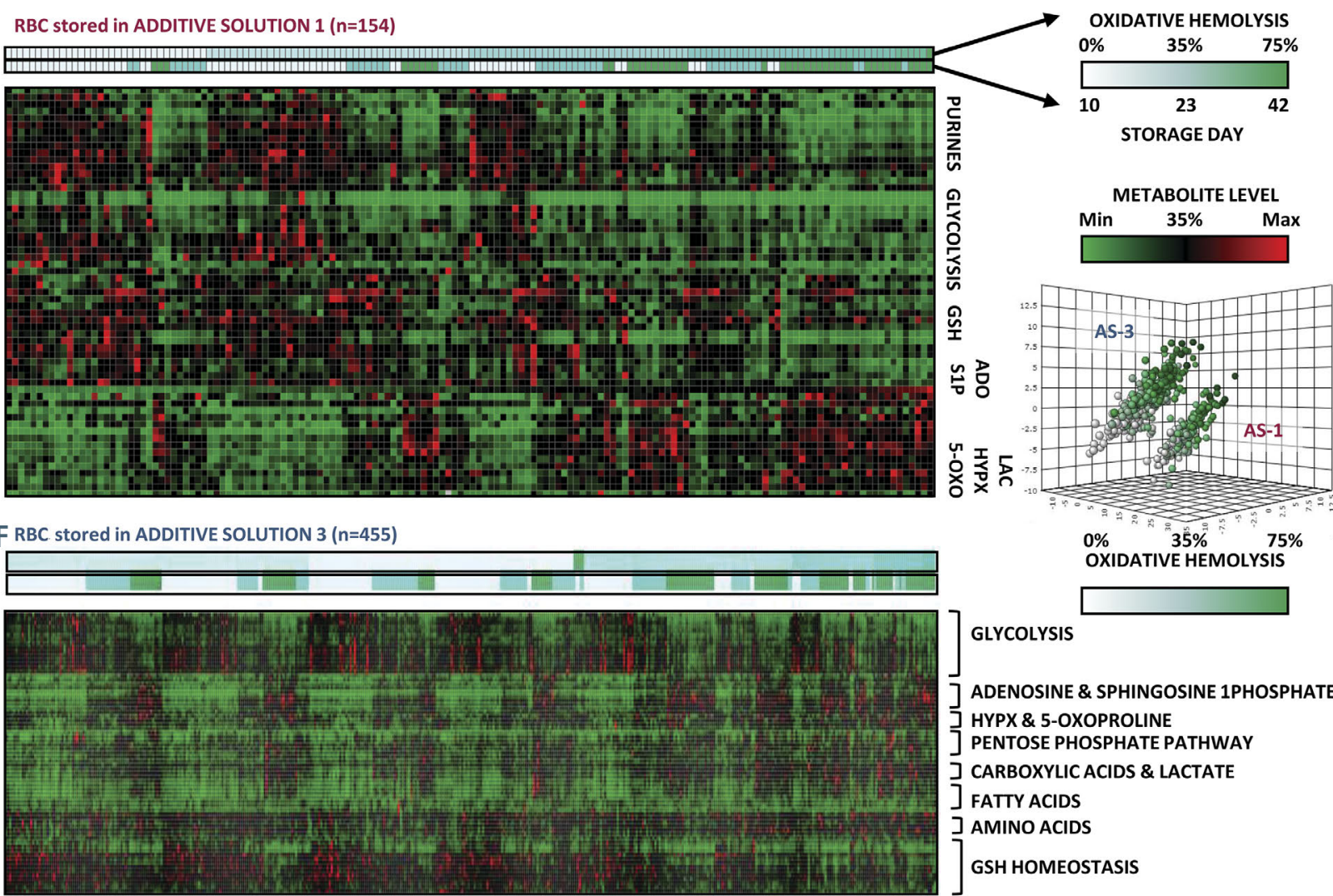

OXIDATIVE HEMOLYSIS

GLYCOLYSIS

] ADENOSINE \& SPHINGOSINE 1PHOSPHATE

HYPX \& 5-OXOPROLINE

] PENTOSE PHOSPHATE PATHWAY

] CARBOXYLIC ACIDS \& LACTATE

] FATTY ACIDS

] AMINO ACIDS

GSH HOMEOSTASIS

Figure 1. Within the framework of the REDS-III Omics study, 13,800 healthy donor volunteers were enrolled and donated a unit of blood, which was stored for 42 days prior to measuring red blood cells (RBC) susceptibility to hemolysis following oxidative insult with AAPH $(A)$. The $5^{\text {th }}$ and $95^{\text {th }}$ extreme hemolyzers $(n=200)$ were recalled and donated a second unit of blood, which was stored for 10, 23 and 42 days prior to testing of oxidative hemolysis (AAPH) and metabolomics (B). Volcano plot representation of metabolic changes at day 42 vs day 10 clearly indicate minimal storage-induced metabolic derangements in RBC from low oxidative hemolyzers (C), while thousands of significant changes were observed in RBC from high oxidative hemolyzers (D). Top metabolic changes positively (red) and negatively (green) correlating (Spearman correlation) with oxidative hemolysis (D) were determined and graphed as heat maps, as a function of oxidative hemolysis and storage day (white to green), storage additives AS-1 (E) vs. AS-3 (F) - a key variable identified by principal component analysis - box on the right), relative abundance (low to high = green to red). Abbreviations: 5-OXO: 5-oxoproline; ADO: adenosine; GSH: reduced glutathione; HYPX: hypoxanthine; LAC: lactate; LogFC: -log(2) fold-change; ROS: reactive oxygen species; S1P: sphingosine 1-phosphate; AAPH: 2,2'-azobis-2-methyl-propanimidamide dihydrochloride. 


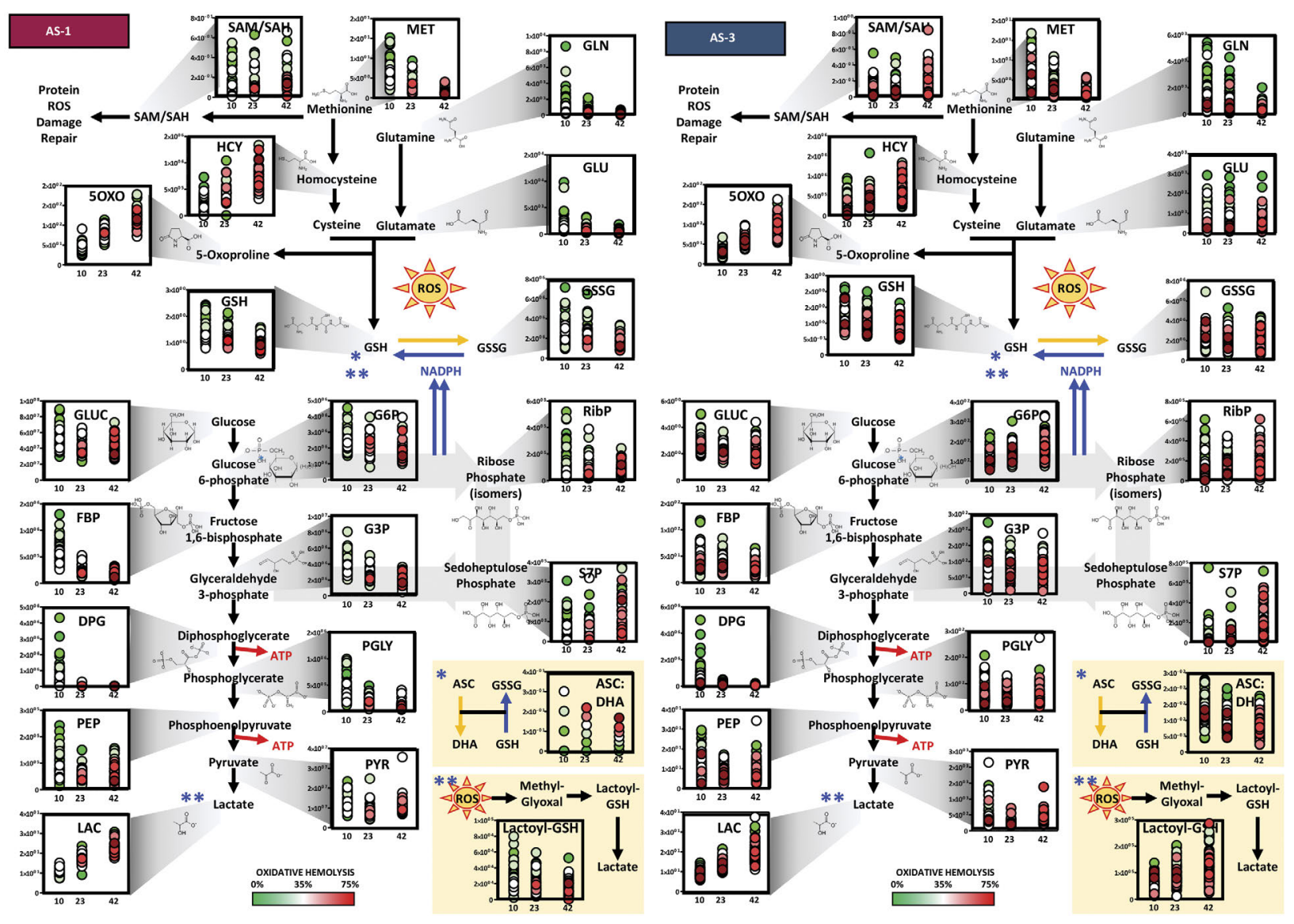

Figure 2. Metabolic changes in glycolysis, pentose phosphate pathway and glutathione homeostasis as a function of storage additives (AS-1 - left; AS-3 - right). On the $x$ axis of each graph, storage day 10, 23 and 42 are represented. Each dot represents an independent measurement and colors are proportional to the oxidative hemolysis measurement for the same sample (green to red = low to high oxidative hemolysis). 5-0XO: 5-oxoproline; ASC:DHA: ascorbate to dehydroascorbate ratios; DPG: 2,3-diphosphoglycerate; FBP: fructose bisphosphate; HCY: homocysteine; G3P: glyceraldehyde 3-phosphate; G6P: glucose 6-phosphate; GLN: glutamine; GLU: glutamate; GLUC: glucose; GSH: reduced glutathione; GSSG: oxidized glutathione; LAC: lactate; LAC-GSH: lactoyl-glutathione; MET: methionine; PEP: phosphoenolpyruvate; PGLY: phosphoglycerate; PYR: pyruvate; RibP: ribosome phosphate (isomers); SAM/SAH: S-adenosylmethionine to S-adenosylhomocysteine ratios; S7P: sedoheptulose phosphate.

\section{Metabolomics and statistical analyses}

These analyses were performed as described ${ }^{36}$ and are extensively reported in the Online Supplementary Methods.

\section{Results}

\section{Red blood cells from donors with low oxidative} hemolysis are less impacted by the metabolic storage lesion

An overview of the experimental design for the pilot study and the metabolomics study on recalled donors is provided in Figure 1A-B. Through a combination of targeted and untargeted analyses, ${ }^{36}$ a total of 19,264 unique chemical formulae were monitored and quantified, out of which 363 metabolites were manually validated against in-house available standards. Absolute quantitation of 50 oxylipids and 45 hydrophilic metabolites including previously identified markers of the RBC metabolic age ${ }^{6}$ - were determined against stable isotopelabeled internal standards. Metabolites were identified at the intact mass and fragmentation (MS/MS) level, as exemplified by reduced GSH - a metabolite critical to RBC redox homeostasis - in the Online Supplementary Figure S1. Volcano plot analyses revealed minimum stor- age-induced metabolic changes at day 42 versus day 10 in $\mathrm{RBC}$ from low oxidative hemolysers, while thousands of significant changes were observed in end of storage RBC from high oxidative hemolysers when compared to day 10 stored RBC (Figure 1C).

Metabolite levels were thus correlated to oxidative hemolysis measurements, i.e., the propensity to hemolyse upon oxidant challenge with AAPH (Online Supplementary Table S1). Metabolites were sorted from positive (red) to negative correlations (green - Spearman correlation) with oxidative hemolysis (Figure 1D; Online Supplementary Table S1). In order to facilitate the visualization of the data, results were plotted as heat maps, as a function of oxidative hemolysis and storage day (white to green), storage additives (AS-1 vs. AS-3, a previously appreciated ${ }^{36}$ key variable identified by principal component analysis - indent on the right), and relative abundance (low to high $=$ green to red). A positive correlation was noted between storage duration and oxidative hemolysis, though significant variability was noted across donors, with some day 23 and day 42 donor samples with low oxidant hemolysis clustering with day 10 $\mathrm{RBC}$, while some donor RBC at day 10 clustered with end of storage RBC from low oxidant donors (Figure 1E). This analysis allowed us to identify key pathways corre- 
lating with oxidant hemolysis, including glycolytic metabolites, metabolites involved in the PPP and GSH homeostasis (Figure 2), purine metabolism and oxidation, arginine and proline metabolism, fatty acids and carboxylic acids (Online Supplementary Figure S2).
Based on these data, we identified the distribution of oxidative hemolysis across all the 599 samples tested in this study and determined the thresholds that discriminated the $33^{\text {rd }}$ and $66^{\text {th }}$ percentiles for this parameter. Based on these thresholds, we generated Receiver
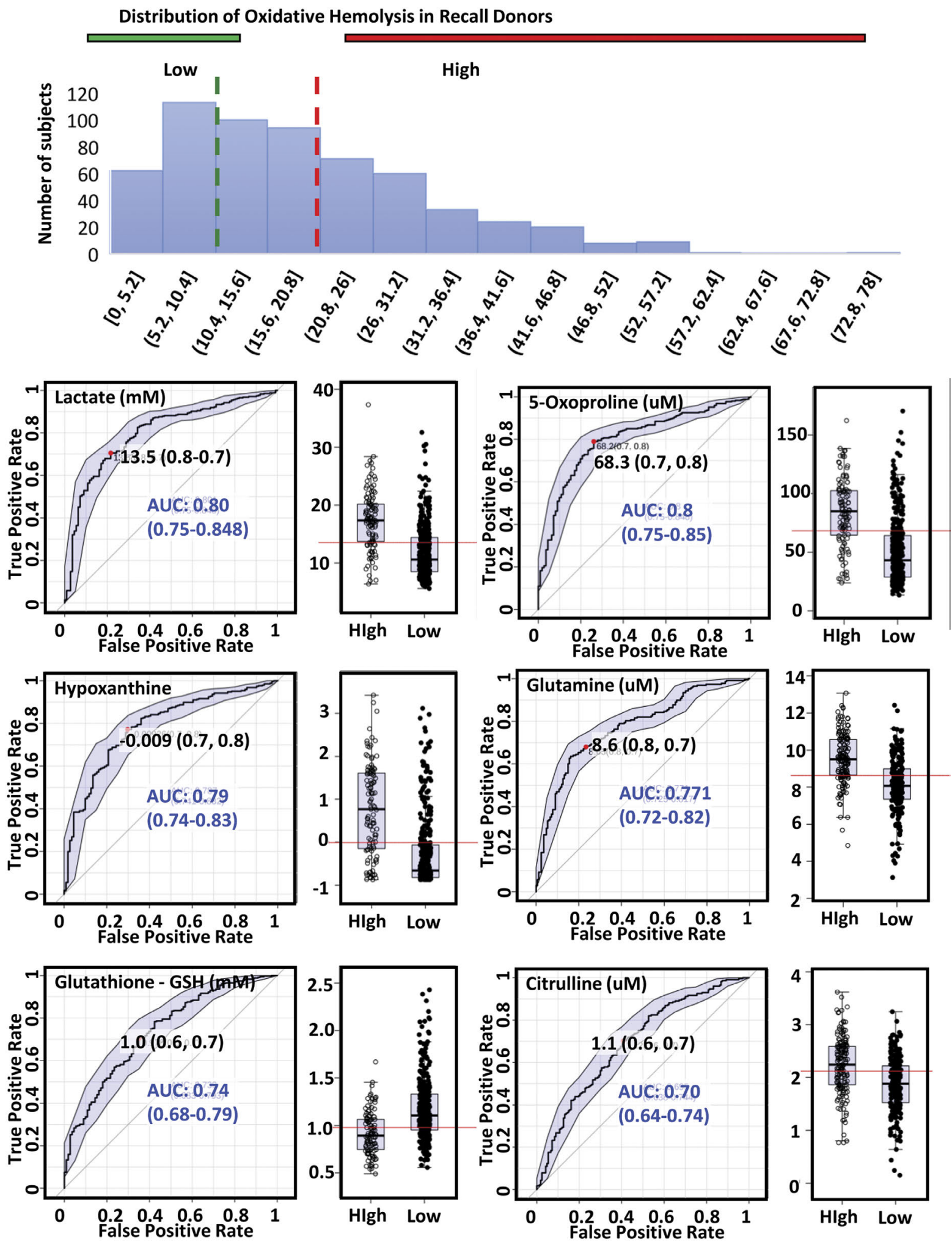

Figure 3. Receiver Operating Characteristic (ROC) curves of metabolic predictors of low and high ( $33^{\text {rd }}$ and $66^{\text {th }}$ percentile) oxidative hemolysis across the recalled donor population. Specificity, sensitivity and absolute quantitative threshold for each metabolite are noted. AUC: area under the curve. 
Operating Characteristic (ROC) curves of metabolic predictors of low and high oxidative hemolysis across the recalled donor population (Figure 3). ROC curves, sensitivities specificities and absolute quantitative thresholds for each metabolite are presented in Figure 3. Of note, concentrations of lactate above $13.5 \mathrm{mM}, 5$-oxoproline (above $68.3 \mathrm{uM})$, glutamine (8.6 uM), GSH (1.0 mM) and citrulline (1.1 uM) had areas under the curve (AUC) $\geq 0.7$.
Oxidative hemolysis is subject to biological variability across donors

As noted above, end of storage RBC from some donors had similar metabolic phenotypes and oxidative hemolysis to day $10 \mathrm{RBC}$ from other donors and vice versa. In this view, we hypothesized that factors other than storage duration alone may impact RBC oxidative hemolysis and the associated metabolic phenotypes. Consistently, we noted
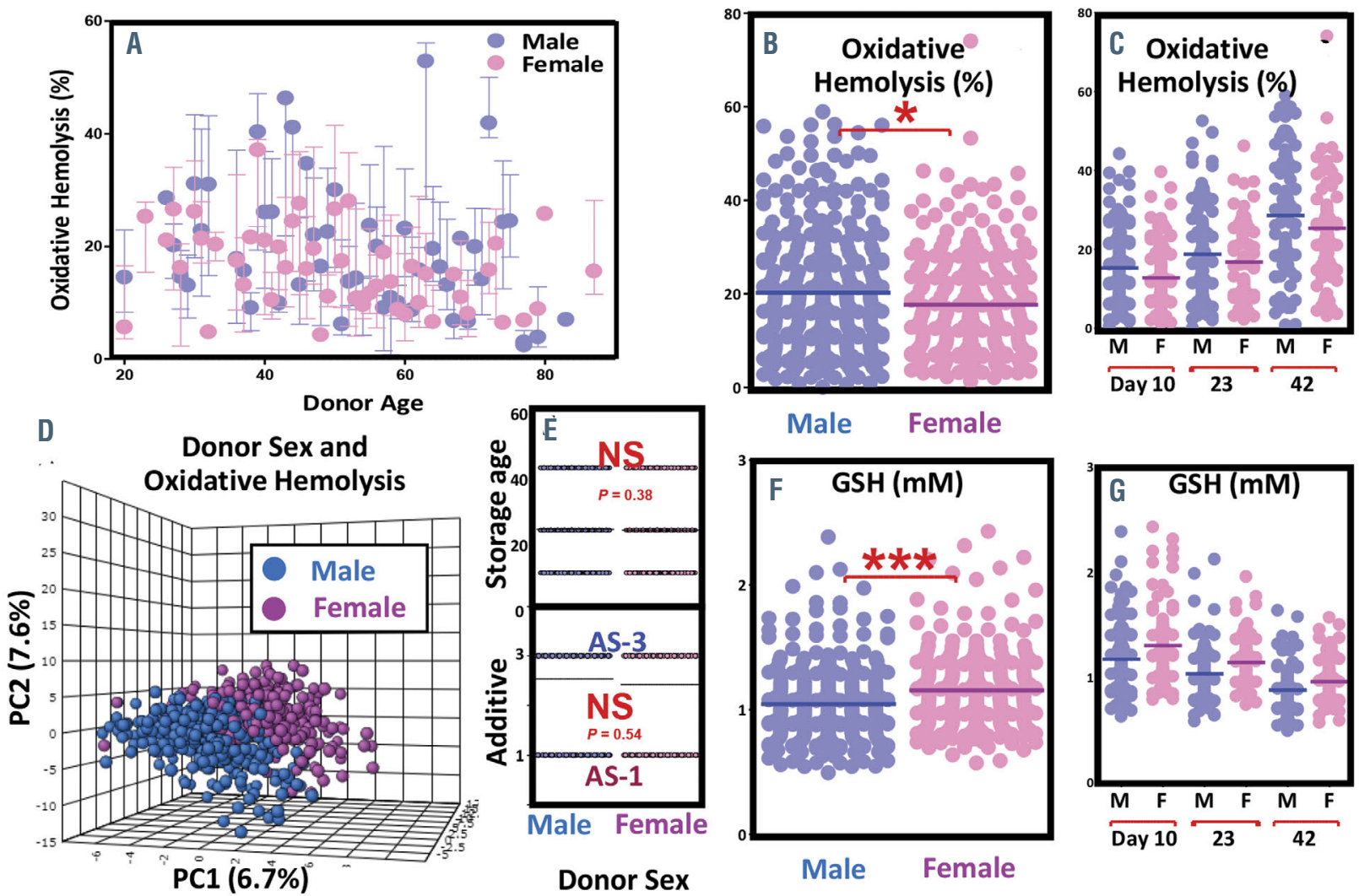

\section{Male Female}
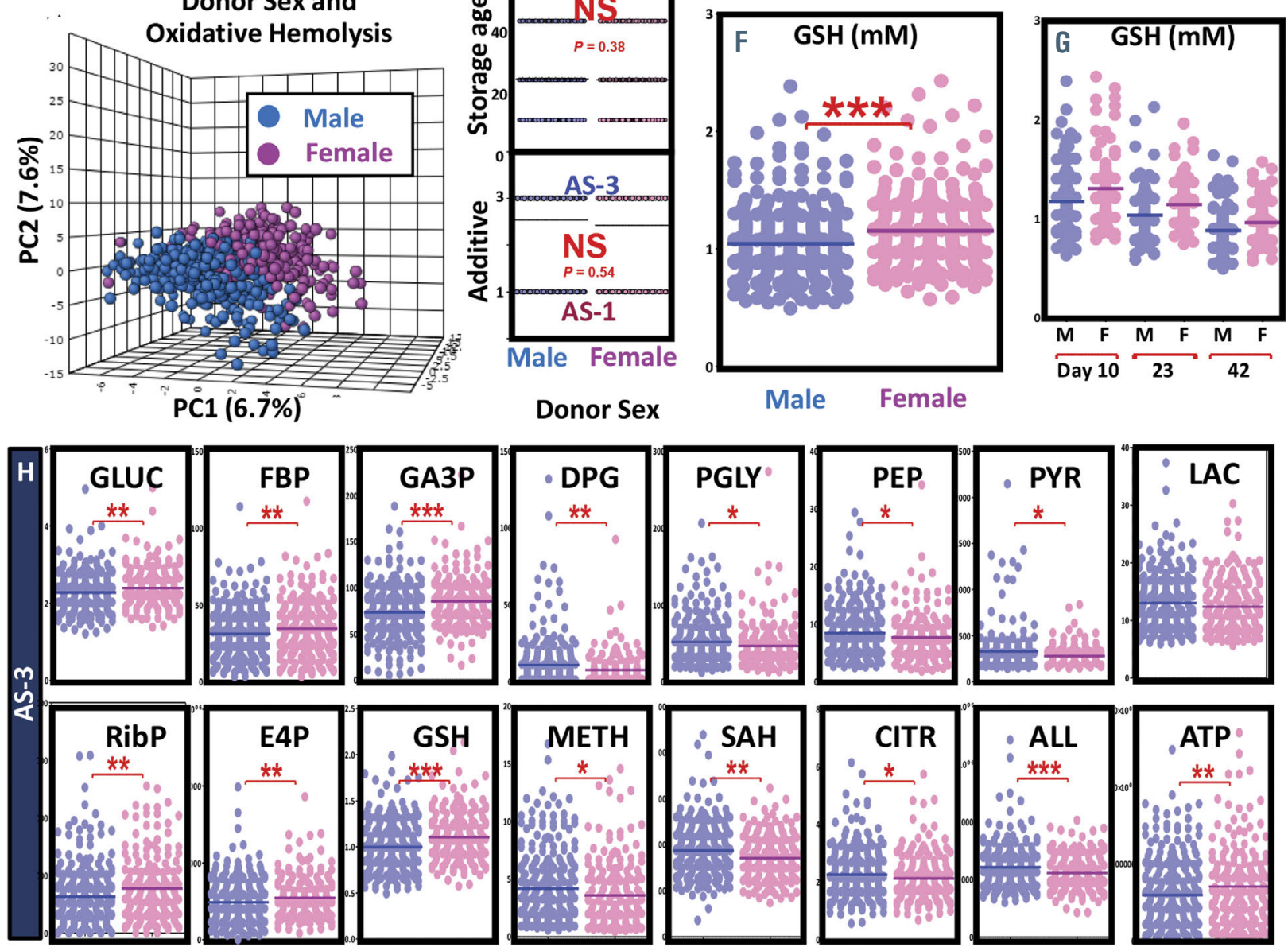

Figure 4. Oxidative hemolysis as a function of donor sex (male vs. female = blue vs. pink) and age (A). Oxidative hemolysis was higher in male donors at any given storage day (B) or storage day 10, 23 and 42 (C). Vice versa, reduced glutathione (GSH) was lower in male donors at any given storage day (F) and in the longitudinal analysis at day 10, 23 and $42(G)$. Partial least squares discriminant analysis (PLS-DA) discriminated between male and female donors (D), in the absence of significant enrichment for AS-3 or AS-1 sample in either group (E). Red blood cells (RBC) from male donors have altered glycolysis, lower activation of the pentose phosphate pathway, altered methionine and citrulline metabolism (samples are plotted with no distinction of storage days, only samples stored in AS-3 are shown in this panel - H). ALL: allantoin; ATP: adenosine triphosphate; CITR: citrate; DPG: 2,3-diphosphoglycerate; E4P: erythrose phosphate; FBP: fructose bisphosphate; GA3P: glyceraldehyde 3-phosphate; GLUC: glucose; GSH: reduced glutathione; LAC: lactate; METH: methionine; PEP: phosphoenolpyruvate; PGLY: phosphoglycerate; PYR: pyruvate; RibP: ribose phosphate (isomers); SAH: S-adenosylhomocysteine. 
that variables such as donor sex, age, ethnicity and storage additives impacted oxidative hemolysis, with blood from female, over 60 years old, and southern Asian donors whose leucoreduction RBC were stored in additive addtive AS-3 having the lowest oxidative hemolysis, independently of storage duration (Online Supplementary Figure S3). In this view, we elaborated the metabolomics data as a function of these variables and independently of storage duration, while controlling for potential mixed effects of combined variables (Figure 4-5; Online Supplementary Figure S4-5).

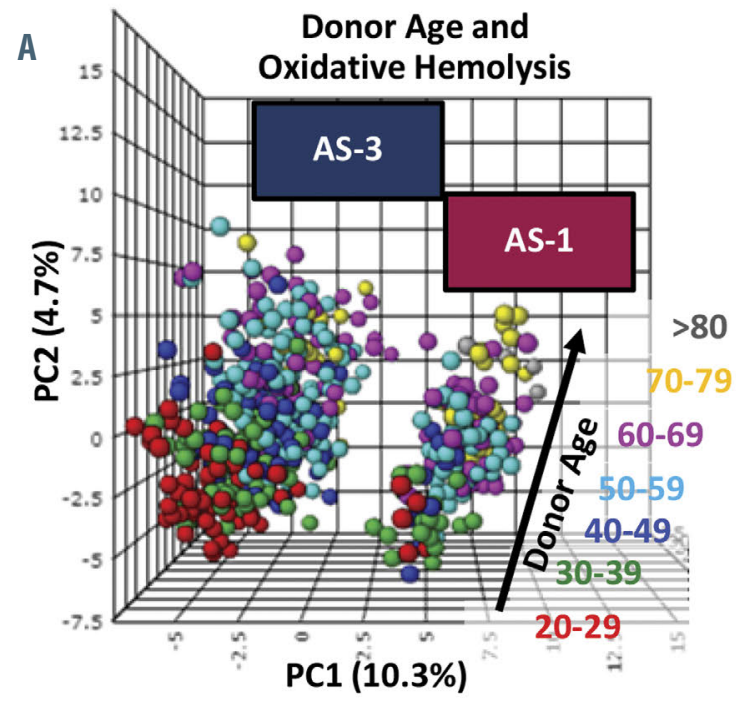

F
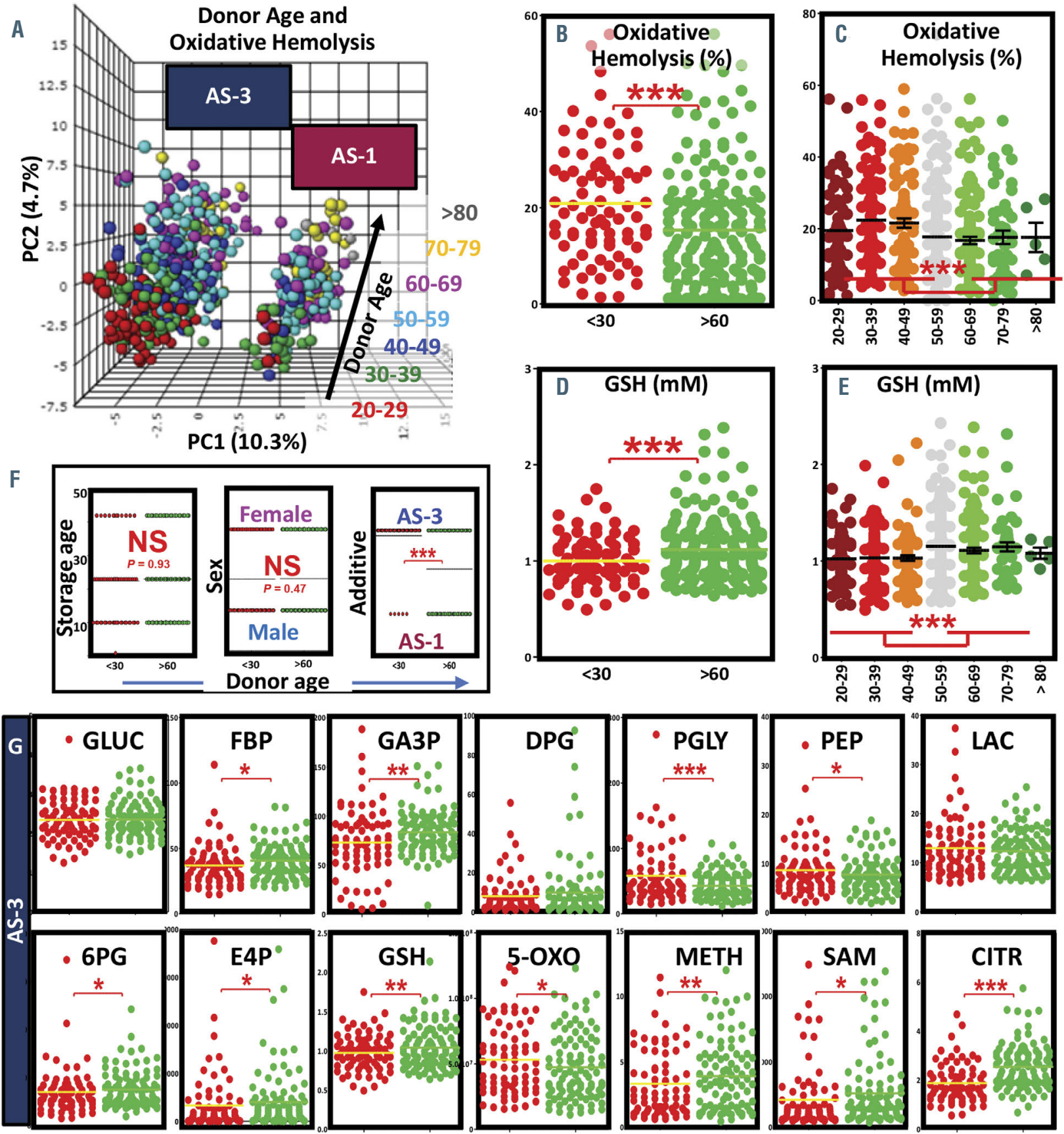

Figure 5. Oxidative hemolysis as a function of donor age (buckets in principal components analysis [PCA] indicate increments of age by decade across second principal component [PC2]). (A). Oxidative hemolysis and reduced glutathione (GSH - donor under 30 years of age vs. over 60 - B-D, respectively; breakdown by decades - C-E) are higher and lower, respectively in red blood cells (RBS) from donors younger than 30 at any given storage day. In F, RBC from donors over 60 vs. under 30 were not significantly different with respect to storage age or donor sex, though the number of RBC from older donors that were stored in AS-1 was significantly higher. Even when controlling for storage additive (AS-3 only - G), RBC from young donors have altered glycolysis, lower levels of pentose phosphate pathway metabolites, altered methionine and citrulline metabolism (samples are plotted with no distinction of storage days). 5-OXO: 5-oxoproline; 6PG: 6-phosphogluconate; CITR: citrate; DPG: 2,3-diphosphoglycerate; E4P: erythrose phosphate; FBP: fructose bisphosphate; GA3P: glyceraldehyde 3-phosphate; GLUC: glucose; GSH: reduced glutathione; LAC: lactate; METH: methionine; NS: not-significant; PEP: phosphoenolpyruvate; PGLY: phosphoglycerate; SAM: S-adenosylmethionine. 
Red blood cells from female and over 60 donors have lower levels of oxidative hemolysis and increased energy and antioxidant metabolism

Following the observations by Kanias and colleagues in the original REDS-III cohort of donors, in the subpopulation of recalled donors (that included the extreme low and high hemolysers from phase I) we noted that RBC from female donors had lower oxidative hemolysis than male counterparts, especially in the age window between 30 and 60 years of age (Figure 4A). This observation held true independently of storage duration (Figure 4B) and at any given storage day tested in this study (Figure 4C). Principal component analysis noted that donor sex explained $6.7 \%$ of the total metabolic heterogeneity across donors (Figure 4D), in the absence of any measurable confounders related to storage duration, storage additive or donor age/ethnicity between the two groups (Figure 4E). GSH levels were significantly higher in RBC from female donors at any given time point tested in the study (Figure 4F-G). This observation was in part explained by increased levels of early glycolytic metabolites in RBC from female donors (independently of storage day), suggestive of a potential switch from glycolysis towards the PPP to generate reducing equivalents - as supported by higher levels of PPP intermediates and endproducts in this group (Figure $4 \mathrm{H}$ ). This metabolic rewiring was accompanied by higher ATP but lower DPG levels, and overall lower methionine and S-adenosylho-
A

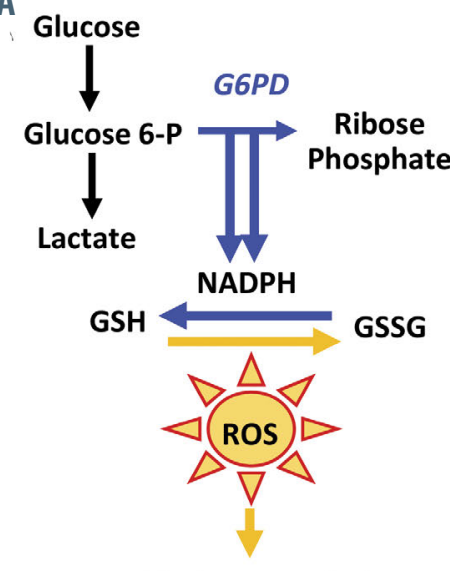

Oxidative Hemolysis

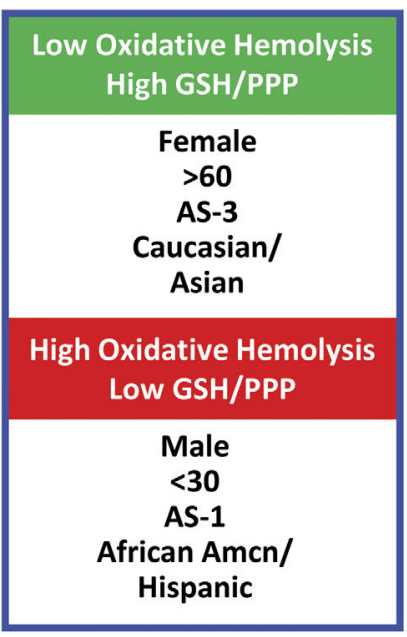

B

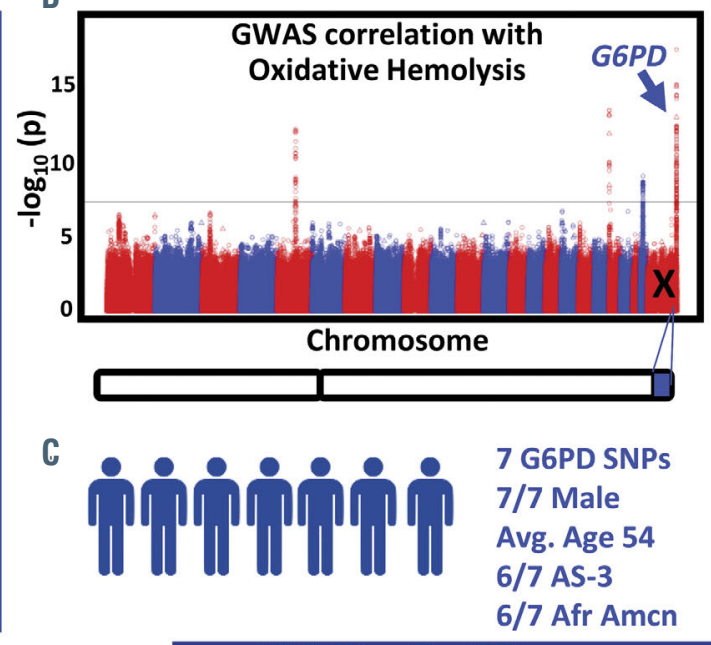

D
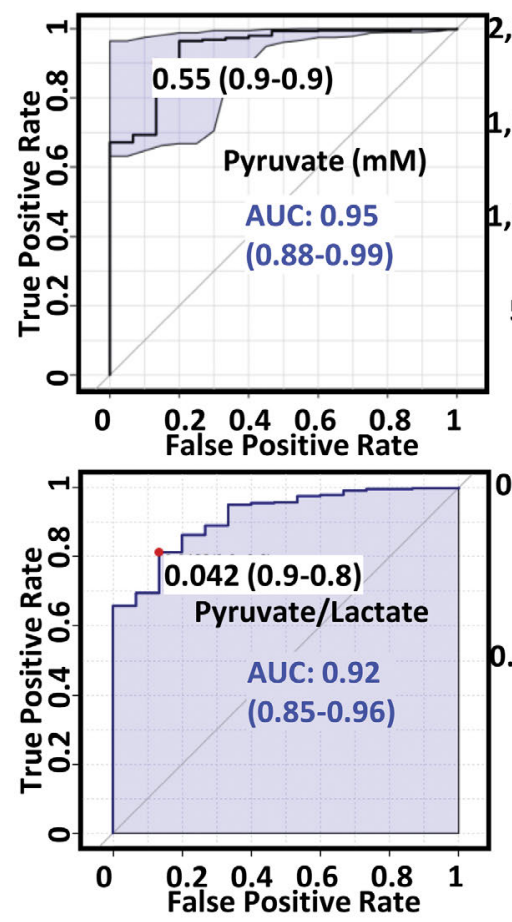
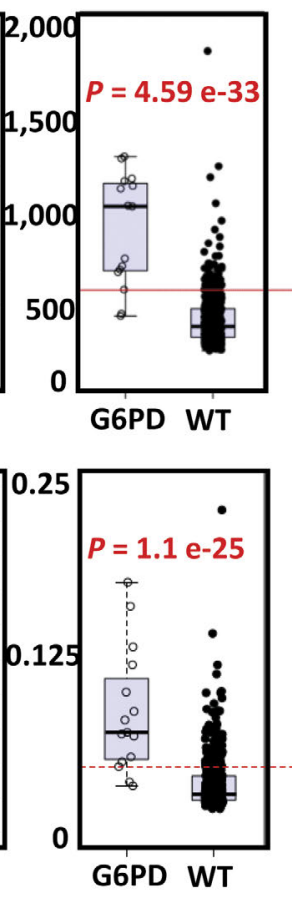
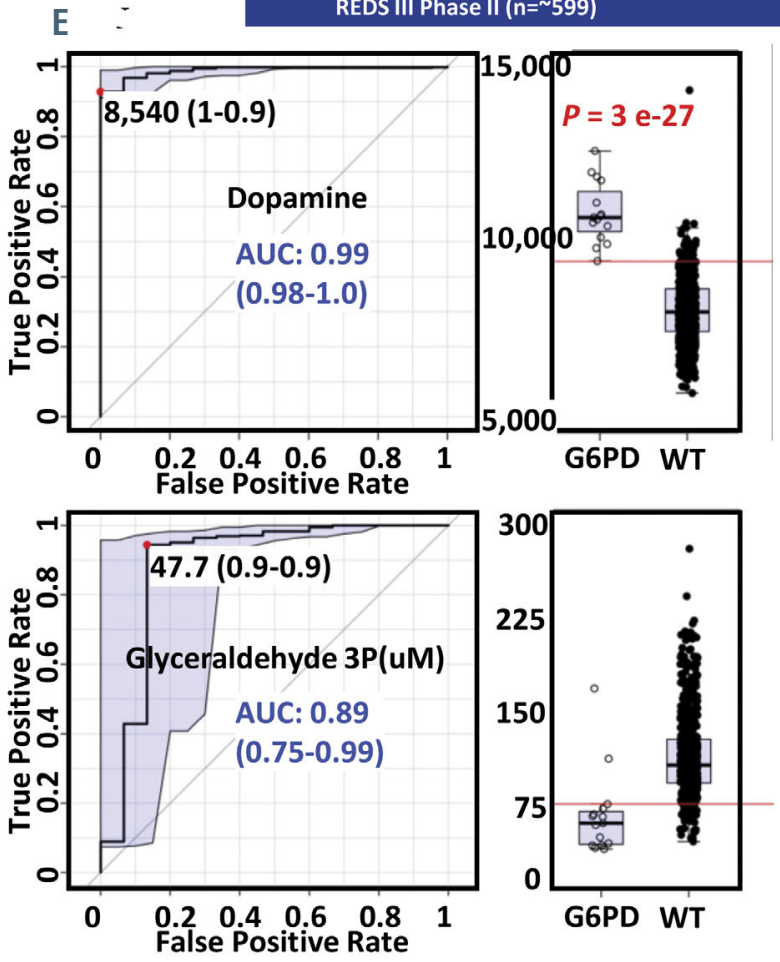

Figure 6. Glucose 6-phosphate dehydrogenase (G6PD) deficiency (A) is a critical factor influencing oxidative hemolsysis. Genome wide association study analysis (GWAS) (B) in a subset of the recalled donor population in the REDS-III study (C). Biomarker analysis of metabolomics data identifies pyruvate and pyruvate/lactate ratios (D), dopamine and glyceraldehyde 3-phosphate (E) as a potential predictor of G6PD deficiency. G6PD: glucose 6-phosphate dehydrogenase; GSH: reduced glutathione; GSSG: oxidized glutathione; NADPH: nicotinamide adenine dinucleotide phosphate, reduced; WT: non-G6PD deficient. AUC: area under the curve. 

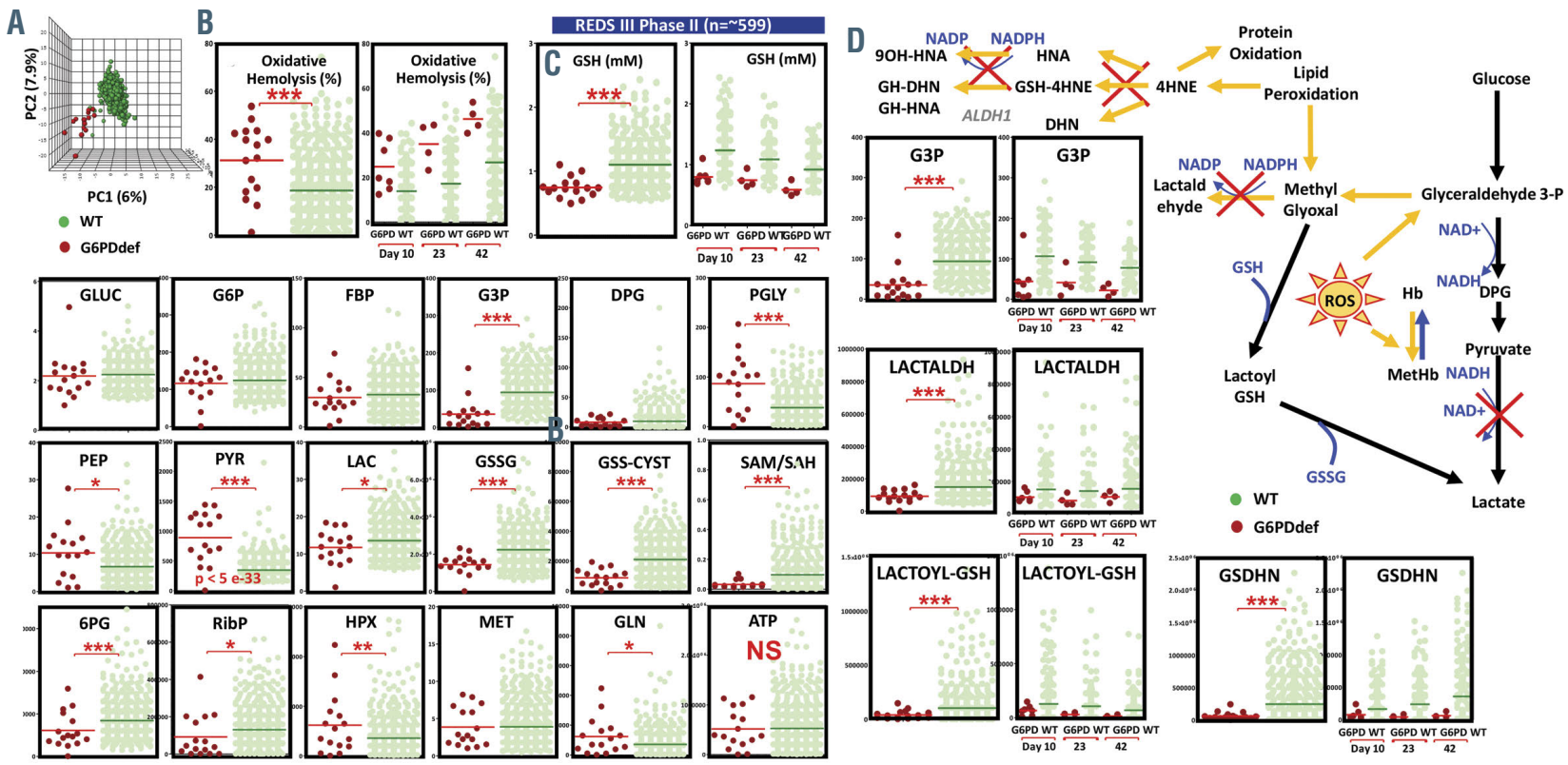

Figure 7. Glucose 6-phosphate dehydrogenase (G6PD) deficient donor from the REDS-III Omics study (A) had higher oxidative hemolysis (B), lower glutathione and lower pentose phosphate pathway activation than G6PD sufficient donors (C). By limiting the availability of reduced glutathione (GSH) and NADPH, G6PD deficiency negatively impact recycling of peroxidized lipids through the glyoxalase pathway and aldehyde dehydrogenase 1 (ALDH1)-dependent steps (D). 4HNE: 4-hydroxynonenal; 6PG: 6-phosphogluconate; ALDH1: aldehyde dehydrogenase 1; ATP: adenosine triphosphate; DPG: 2,3-diphosphoglycerate; E4P: erythrose phosphate; FBP: fructose bisphosphate; G3P: glyceraldehyde 3-phosphate; GLN: glutamine; GLUC: glucose; GSDHN: glutathionyl dihydroxynonenal; GSH: reduced glutathione; HNA: hydroxynonenoic acid; HPX: hypoxanthine; LAC: lactate; LACTALDH: lactaldehyde; METH: methionine; PEP: phosphoenolpyruvate; PGLY: phosphoglycerate; RibP: ribose phosphate (isomers); SAM/SAH: S-adenosylmethionine to S-Adenosylhomocysteine ratios;

mocysteine (SAH) levels, suggestive of decreased basal oxidant stress in female RBC.

Similarly, donor age explained $\sim 5 \%$ of the total metabolic variance across donors (second principal component [PC2] in the principal component analysis in Figure 5A). Unexpectedly, RBC from young donors had higher oxidative hemolysis (Figure 5B-C) and lower levels of GSH (Figure 5D), which was accompanied by altered glycolysis, lower activation of the PPP, altered methionine and citrulline metabolism independently from storage duration (Figure 5).

\section{Correlations of oxidative hemolysis and energy and antioxidant metabolism relative to storage additives and donor race-ethnicities}

Expanding on prior work, ${ }^{36}$ we confirmed a significant impact $(\sim 11 \%)$ of storage additives on the metabolic heterogeneity of the 599 tested units, which was here correlated to decreased oxidative hemolysis in storage additive AS-3 RBC (Online Supplementary Figure S4A-C). Lower oxidative hemolysis was accompanied by higher levels of GSH in AS-3 RBC, faster glycolysis, higher ATP and DPG, higher PPP, decreased purine oxidation and protein damage-repair pathway activity (SAH) in AS-3 RBC independently of storage duration (Online Supplementary Figure S4D$E)$. Donor ethnicity had a significant impact on oxidative hemolysis as well ( $9.2 \%$ of the total variance), with RBC from Hispanic/Latino donors having significantly higher oxidative hemolysis than RBC from Asian and Southern Asian donors (Online Supplementary Figure S5). Of note, $\mathrm{RBC}$ from African American donors had high levels of phosphoglycerate and ribose phosphate, methionine and arginine in comparison to RBC from Asian donors.

\section{Altered glutathione metabolism and recycling underlying increases in oxidative hemolysis may be explained by glucose 6-phosphate dehydrogenase status}

G6PD is the rate-limiting enzyme of the PPP, a key antioxidant pathway in the mature erythrocyte for the recycling of oxidized GSH (Figure 6A). Within the framework of the RBC-Omics genome wide association study (GWAS), a specific single nucleotide polymorphisms (SNP) to G6PD was found to correlate significantly to oxidative hemolysis (GWAS analysis - Figure 6B). Such polymorphism, better described in Page et al. (under review), include point mutations resulting from SNP on chromosome X, rs1050828 (ASN126ASP) which is common in individuals of African descent (hence named G6PD A-variant). This finding was expected, in that the oxidative hemolysis test used to screen the donors in the present study is similar to the clinical test for G6PD deficiency in the clinical setting. Among the subjects tested in the present study, we identified SNP in G6PD potentially associated with oxidative hemolysis in the seven recalled samples that underwent metabolomics in the present study (Figure 6C). Bioinformatics integration of the top 100 hits from the GWAS and metabolomics analysis against oxidative hemolysis was performed via OmicsNet; elaborations include a gene-centric (top) and metabolitecentric (bottom) network view, both analyses confirming a central role for G6PD in mediating energy and redox homeostasis (Online Supplementary Figure 6D). This unsupervised analysis identified the centrality of the G6PD network and branching pathways (including NADPH- and GSH-dependent pathways) in the REDS-III omics recalled donor cohort. We thus used the metabolomics data to per- 
form a biomarker analysis of the whole population versus the seven subjects carrying G6PD A- SNP. This analysis highlighted pyruvate (G6PD deficient if pyruvate $<0.546$; AUC: 0.95 ; $P$-value $=4.59$ e-33), pyruvate/lactate ratios and dopamine (whose metabolism is NADPH-dependent) and glyceraldehyde 3-phosphate (whose metabolism is $\mathrm{NADH}$-dependent) as the top markers of G6PD deficiency in this study (Figure 6D-E).

Putative G6PD deficient donors were indeed characterized by significant increases in oxidative hemolysis and decreases in GSH (Figure 7). Even though G6PD deficiency was inferred from the SNP detected in the GWAS data, metabolomics analyses confirmed that RBC from the donors bearing those SNP were indeed characterized by an ablation of the PPP and complete depletion of the total glutathione pool, including GSH but also its oxidized form, GSH-adducts (Figure 7A). By limiting the availability of reduced GSH and NADPH, G6PD deficiency negatively impacts recycling of peroxidized lipids through the glyoxalase pathway and aldehyde dehydrogenase 1 (ALDH1)-dependent steps (Figure 7D). Significantly, decreases were indeed observed in the levels of metabolites that from pathways requiring either/both NADPH and GSH for the detoxification of oxidized lipids (Figure 7D), including lactaldehyde, lactoyl-glutathione, glutathionyl- dihydroxynonane (GSDHN).

\section{Discussion}

The mature erythrocyte has evolved to maximize its capacity to carry and deliver oxygen to tissues. As such, RBC are packed with $\sim 270$ million copies of hemoglobin per cell and $\sim 66 \%$ of the total iron in the human body. When in the presence of oxygen (e.g., oxygen saturation rises above $90 \%$ just after 3 weeks of storage owing to the rapid consumption of DPG22), iron can trigger Fenton reactions that generate reactive oxygen species. These phenomena are largely impacted by post-donation hemoglobin-oxygen saturation, which has been noted to vary widely across healthy blood donors. ${ }^{13,21}$ While RBC have also evolved strategies to cope with such oxidant stress, the propensity of RBC to hemolyze following oxidative injury has recently been noted to depend on donor demographics, including donor sex, age and race-ethnicity. ${ }^{33}$ Understanding the etiological contributors to such donordependent variance might shed light not just on RBC storage biology, but also contribute significantly to our understanding of those pathologies associated with RBC hemolysis due to exacerbated oxidant stress (e.g., sickle cell disease, $\beta$-thalassemia, pulmonary hypertension, Down syndrome, inflammaging ${ }^{39}$ ). In the present study, we sought to investigate the metabolic underpinnings of $\mathrm{RBC}$ susceptibility to oxidant stress-induced hemolysis at the end of storage.

This study challenges the dogma that the "age of blood" is the main factor impacting RBC storability and that the progressive accumulation of the storage lesion inevitably results in poor quality blood across all donors by the end of the shelf-life of the stored unit. While donor biology, including sex, age and ethnicity, had been previously identified as determinants of RBC susceptibility to hemolysis following oxidant insults, ${ }^{33}$ by leveraging state of the art metabolomics and lipidomics tools, we report for the first time that these observations could be explained by metabolic factors in the RBC from these donors. Specifically, donors who are less susceptible to oxidant hemolysis (e.g., female donors, donors over 60 years of age and donors of Asian/South Asian race-ethnicity, and leucoreduction RBC components preserved in AS-3) are characterized by higher levels of reduced GSH - independently of the storage duration - higher activation of the PPP and improved preservation of the protein damage-repair mechanisms that rely on methionine metabolism (and intertwined arginine metabolism).

Caution is advised in the interpretation of the presented results, in that (i) it is unclear whether oxidative hemolysis is predictive of clinically-relevant outcomes and (ii) RBC that are less susceptible to oxidant hemolysis may on the other hand be more susceptible to lysis following mechanical and osmotic insults. In this view, the biomarkers and biological correlates to oxidative hemolysis identified here are relevant and potentially translatable to studies pertaining oxidant injury, though they may be less relevant for studies on RBC function under mechanical or osmotic stress, such as for example in the capillaries and in the kidney. Future studies will investigate metabolic correlates and potential mechanistic determinants to these phenotypes. However, prior work has highlighted the clinical relevance of conditions that are associated with increased oxidative hemolysis, such as a causative link between G6PD deficiency and decreases in end of storage autologous post-transfusion recovery in healthy volunteers ${ }^{30}$ and post-transfusion circulation in sickle cell recipients. ${ }^{29}$

Previous studies had shown that RBC from G6PD deficient donors (the most common enzymopathy in humans, affecting $\sim 10 \%$ of the donor population in some metropolitan areas ${ }^{26}$ ) are naturally incapable of coping with oxidant stress owing to the ablation of the NADPHgenerating PPP. As a result, RBC from G6PD deficient donors favor energy metabolism over antioxidant pathways, which protects them from morphological changes but makes them more susceptible to oxidant insults upon storage. ${ }^{28}$ This observation has potential clinical implications, in that transfusion of blood from G6PD deficient donors into recipients suffering from elevated oxidative stress (e.g., sickle cell recipients ${ }^{40}$ ) may be suboptimal and could potentially promote untoward transfusion consequences or, at least, decrease the dose-efficacy of transfusion therapies. In the present study, by combining metabolomics and genomics data from the RBC-Omics study (Page et al. under review and Guo et al. ${ }^{41}$ ), we identified a key role of antioxidant systems that rely on reducing equivalents such as GSH and NADPH to cope with oxidant stress - such as G6PD deficiency. While this result was largerly expected, the present study advanced our understanding of G6PD deficiency by providing genetic and metabolomic metabolic markers (e.g., pyruvate/lactate ratios, absolute concentration of pyruvate) that complement spectrophotometric assays of the enzymatic activity of G6PD. ${ }^{42}$ Some markers like pyruvate to lactate ratio were previously associated with increased activation of $\mathrm{NADH}$-dependent methemoglobin reductase. ${ }^{28}$ As such, since glyceraldehyde 3-phosphate metabolism is dependent on $\mathrm{NAD}+\mathrm{NADH}$ ratios, the two markers observed here are metabolically linked. On the other hand, dopamine metabolism is NADPH-dependent - suggesting a role for monoamine oxidase activity in the context of RBC metabolism and storability as a function 
of oxidant stress. Of note, this observation in parts reconciles the impact of donor sex, age and ethnicity on the propensity to hemolyse following oxidant insult. Indeed, G6PD is coded by a gene on chromosome $\mathrm{X}$, which makes female donors - who carry two copies of this chromosome - less likely to be fully deficient.

On the other hand, G6PD activity declines with the RBC age and human aging, ${ }^{43,44}$ which makes our present findings counterintuitive, since higher levels of reduced GSH and improved redox homeostasis were observed in RBC from old donors. A simple explanation may be due to a sex (male) and ethnicity bias of our younger recall donor population in this study. Alternatively, it has been previously shown that RBC lifespans are shorter in older subjects..$^{45}$ Though merely speculative at this stage, a potential alternative explanation to the observed lower oxidative hemolysis in female and older donors may be associated with alterations to iron metabolism, including for example iron deficiency or decreased ferritin levels in blood from older, female donors. This would in turn limit the availability of iron, a key substrate for radical-generating reactions. Further studies are currently in process to test blood ferritin levels in this cohort. ${ }^{46}$ In the meantime, it has been noted that frequent blood donors in this cohort tended to be white, older age ( $56+5$ years) male who were characterized by lower oxidative hemolysis. ${ }^{47}$ Alternatively, it could be hypothesized that RBC from older or pre-menopausal female donors are either exposed to higher basal levels of stress or more rapidly renewed in the bloodstream (owing to either a shorter physiological lifespan in the former group or because of other sex-related confounders in the latter). Further studies will be necessary to identify the impact of stress erythropoiesis on the maturation of erythrocytes in a background of inflammation in aging ${ }^{48}$ or as a function of donor sex and sex-defining hormones. ${ }^{32}$

The use of metabolomics to complement existing genomics information from the RBC-Omics study allowed us to identify as of yet unexplored pathways with respect to blood storage and the storage lesion. Of note, several oxylipins - markers of post-transfusion recovery in murine models ${ }^{14}$ - were amongst the top positive correlates of oxidative hemolysis. Donors with high oxidative hemolysis, including G6PD deficient donors an extreme subgroup of these high hemolysers - were characterized by a decreased capacity to detoxify lipids through aldehyde dehydrogenase 1-dependent reactions. Of note, ALDH1 activity depends upon NADPH availability, in like fashion to the ferroreductase STEAP 3 - a genetic determinant of the heterogeneity of post-transfusion recovery across mouse strains. ${ }^{14}$

In a recent assessment of intradonor hemolytic propensity within the framework of the RBC-Omics study, Lanteri and colleagues have reported that oxidative hemolysis has a strong donor-dependency across multiple donations ${ }^{35}$ However, the authors also noted that oxidative hemolysis was less reproducible across multiple donations than osmotic hemolysis. This observation is in part explained by the impact of environmental factors rather than donor genetics alone on RBC propensity to hemolysis following oxidant insults, as our metabolomics results suggest here, in that we noted significant negative correlations between the levels of dietary antioxidant metabolites (e.g., epigallocatechin and ergothioneine, abundant in tea - Figure 1D) and oxidative hemolysis. If confirmed in mechanistic studies, this observation could pave the way for considering the introduction of dietary supplements prior to donation in recurring donors. While this study focused on donor biology, other factors such as donor habits (e.g., smoking ${ }^{49}$ ) or environmental exposures beyond diet could play a role in the observed hetereogeneity in oxidative hemolysis across donors and the partial longitudinal reproducibility of this parameter in the same donor across multiple donations. ${ }^{35}$ Finally, we anticipate that our findings will be relevant not just to a readership of transfusion medicine experts, but they will also impact those fields where appreciation of RBC propensity to hemolyse as a function of oxidative injury is critical to the pathophysiology of diseases like sickle cell disease, pulmonary hypertension, Parkinson's or Alzheimer's, sepsis or aging. ${ }^{50}$

\section{RBC-Omics study group members}

The NHLBI Recipient Epidemiology Donor Evaluation StudyIII (REDS-III), Red Blood Cell (RBC)-Omics study, is the responsibility of the following persons: Hubs: AE Mast, JL Gottschall, WB, LA, JM, AH, ZU, and VJ, BloodCenter of Wisconsin, Milwaukee, WI, USA; DJT, JEK and PAD'A, The Institute for Transfusion Medicine (ITXM), Pittsburgh, PA, USA; E. L. Murphy and AMG, University of California, San Francisco, San Francisco, CA, USA; RGC, BRS, and STJ; American Red Cross Blood Services, Farmington, CT, USA; Data coordinating center: DJB, MTS, SME, GPP, YG, NH, $D R$, and $B C S$; RTI International, Rockville, MD, USA; Central and testing laboratories: $M P B, M C L, M S$, and $S K$, Blood Systems Research Institute, San Francisco, CA, USA; TK and MG, Pittsburgh Heart, Lung, Blood, and Vascular Medicine Institute, Division of Pulmonary, Allergy and Critical Care Medicine, University of Pittsburgh, Pittsburgh, PA, USA; Steering committee chairman: SHK, University of British Columbia, Victoria, BC, Canada; National Heart, Lung, and Blood Institute, National Institutes of Health: SAG, KBM and $A M C$.

\section{Disclosures}

Though unrelated to the contents of this manuscripts, the authors declare that $A D$ is a founder of Omix Technologies Inc and Altis Biosciencens LLC. James C Zimring serves as a consultant for Rubius Therapeutics. All the other authors disclose no conflicts of interest relevant to this study. Angelo D'Alessandro is a consultant for Hemanext Inc. James C Zimring serves on the scientific advisory board for Rubius Therapeutics. All the other authors disclose no conflicts of interest relevant to this study.

\section{Contributions}

TK, SK, MTG, ML, MS, MPB, GP, JCZ designed the study. AD, XF, JAR, RCH, JCZ performed metabolomics analyses; YG, GP performed genomics analyses; TK performed oxidative hemolysis measurements; $A D$ prepared figures and wrote the first version of the manuscript. All the authors contributed to the finalization of the manuscript.

\section{Acknowledgments}

The content is solely the responsibility of the authors and does not necessarily represent the official views of the National Institutes of Health. The authors would like to express their gratitude Dr. Simone Glynn of NHLBI for her support throughout this study, the RBC-Omics research staff at all participating blood centers and testing labs for their contribution to this project, and to all blood donors who agreed to participate in this study. 


\section{Funding}

Research reported in this publication was funded by the NHLBI Recipient Epidemiology and Donor Evaluation StudyIII (REDS-III), which was supported by NHLBI contracts NHLBI HHSN2682011-00001I, -00002I, -00003I, 00004I, 00005I,-00006I,-00007I,-00008I, and -00009I, as well as funds from the the National Institute of General and Medical Sciences (RM1GM131968 to ADA), NHLBI R01HL146442 and R01HL149714 (ADA), R01HL148151 (ADA, JCZ), the Boettcher Webb-Waring Investigator Award $(A D A)$ and a Shared Instrument grant by the National Institute of Health (S10OD021641).

\section{References}

1. D'Alessandro A, Kriebardis AG, Rinalducci $\mathrm{S}$, et al. An update on red blood cell storage lesions, as gleaned through biochemistry and omics technologies. Transfusion. 2015; 55(1):205-219.

2. Bardyn M, Rappaz B, Jaferzadeh K, et al. Red blood cells ageing markers: a multiparametric analysis. Blood Transfus. 2017;15(3):239-248.

3. Blasi B, D'Alessandro A, Ramundo N, Zolla L. Red blood cell storage and cell morphology. Transfus. Med Oxf Engl. 2012;22(2):9096.

4. Roussel C, Monnier S, Dussiot M, et al. Fluorescence exclusion: a simple method to assess projected surface, volume and morphology of red blood cells stored in blood bank. Front Med. 2018;5:164.

5. Yoshida T, Prudent M, D'alessandro A. Red blood cell storage lesion: causes and potential clinical consequences. Blood Transfus. 2019;17(1):27-52.

6. Paglia G, D'Alessandro A, Rolfsson Ó, et al. Biomarkers defining the metabolic age of red blood cells during cold storage. Blood. 2016;128(13):e43-50.

7. Zimring James C. Widening our gaze of red blood storage haze: a role for metabolomics. Transfusion. 2015;55(6):1139-1142.

8. Yoshida T, Prudent M, D'alessandro A. Red blood cell storage lesion: causes and potential clinical consequences. Blood Transfus. 2019;17(1):27-52.

9. Tsai AG, Hofmann A, Cabrales P, Intaglietta $M$. Perfusion vs. oxygen delivery in transfusion with "fresh" and "old" red blood cells: The experimental evidence. Transfus Apher Sci. 2010;43(1):69-78.

10. Heaton A, Keegan T, Holme S. In vivo regeneration of red cell 2,3-diphosphoglycerate following transfusion of DPG-depleted AS-1, AS-3 and CPDA-1 red cells. Br J Haematol. 1989;71(1):131-136.

11. D'Alessandro A, Reisz JA, Culp-Hill R, et al. Metabolic effect of alkaline additives and guanosine/gluconate in storage solutions for red blood cells. Transfusion. 2018; 58(8):1992-2002.

12. Van 't Erve TJ, Wagner BA, Martin SM, et al. The heritability of hemolysis in stored human red blood cells. Transfusion. 2015; 55(6):1178-1185

13. Nemkov T, Sun K, Reisz JA, et al. Hypoxia modulates the purine salvage pathway and decreases red blood cell and supernatant levels of hypoxanthine during refrigerated storage. Haematologica. 2018;103(2):361-372.

14. Howie HL, Hay AM, de Wolski K, et al. Differences in Steap3 expression are a mechanism of genetic variation of RBC storage and oxidative damage in mice. Blood Adv. 2019;3(15):2272-2285.

15. Belpulsi D, Spitalnik SL, Hod EA. The controversy over the age of blood: what do the clinical trials really teach us? Blood Transfus. 2017;15(2):112-115.

16. Roubinian NH, Westlake M, St Lezin EM, et al. Association of donor age, body mass index, hemoglobin, and smoking status with in-hospital mortality and length of stay among red blood cell-transfused recipients. Transfusion. 2019;59(11):3362-3370.

17. D'Alessandro A. From Omics technologies to personalized transfusion medicine. Expert Rev Proteomics. 2019;16(3):215-225.

18. Koch CG, Duncan AI, Figueroa P, et al. Real age: red blood cell aging during storage. Ann Thorac Surg. 2019;107(3):973-980.

19. Bardyn M, Maye S, Lesch A, et al. The antioxidant capacity of erythrocyte concentrates is increased during the first week of storage and correlated with the uric acid level. Vox Sang. 2017;112(7):638-647.

20. Jordan A, Chen D, Yi Q-L, et al. Assessing the influence of component processing and donor characteristics on quality of red cell concentrates using quality control data. Vox Sang. 2016;111(1):8-15

21. Yoshida T, Blair A, D'alessandro A, et al. Enhancing uniformity and overall quality of red cell concentrate with anaerobic storage. Blood Transfus. 2017;15(2):172-181.

22. Reisz JA, Wither MJ, Dzieciatkowska M, et al. Oxidative modifications of glyceraldehyde 3-phosphate dehydrogenase regulate metabolic reprogramming of stored red blood cells. Blood. 2016;128(12):e32-42.

23. Wither M, Dzieciatkowska M, Nemkov T, et al. Hemoglobin oxidation at functional amino acid residues during routine storage of red blood cells. Transfusion. 2016;56(2):421-426

24. Tzounakas VL, Georgatzakou HT, Kriebardis AG, et al. Uric acid variation among regular blood donors is indicative of red blood cell susceptibility to storage lesion markers: A new hypothesis tested. Transfusion. 2015;55(11):2659-2671.

25. Tzounakas VL, Georgatzakou HT, Kriebardis AG, et al. Donor variation effect on red blood cell storage lesion: a multivariable, yet consistent, story. Transfusion. 2016;56(6):1274-1286.

26.Francis RO, Jhang JS, Pham HP, et al. Glucose-6-phosphate dehydrogenase deficiency in transfusion medicine: the unknown risks. Vox Sang. 2013;105(4):271282.

27 Reisz JA, Tzounakas VL, Nemkov T, et al. Metabolic linkage and correlations to storage capacity in erythrocytes from glucose 6-phosphate dehydrogenase-deficient donors. Front Med. 2017;4:248.

28. Tzounakas VL, Kriebardis AG, Georgatzakou HT, et al. Glucose 6-phosphate dehydrogenase deficient subjects may be better "storers" than donors of red blood cells. Free Radic Biol Med. 2016;96:152-165.

29. Sagiv E, Fasano RM, Luban NLC, et al. Glucose-6-phosphate-dehydrogenase deficient red blood cell units are associated with decreased posttransfusion red blood cell survival in children with sickle cell disease. Am J Hematol. 2018;93(5):630-634

30. Francis RO, D'Alessandro A, Eisenberger A, et al. Donor glucose-6-phosphate dehydrogenase deficiency decreases blood quality for transfusion. J Clin Invest. 2020;130 (5):2270-2285

31. Glucose-6-phosphate dehydrogenase deficiency. WHO Working Group. Bull. World Health Organ. 1989;67(6):601-611.

32. Kanias T, Sinchar D, Osei-Hwedieh D, et al. Testosterone-dependent sex differences in red blood cell hemolysis in storage, stress, and disease. Transfusion. 2016;56(10):25712583.

33. Kanias T, Lanteri MC, Page GP, et al. Ethnicity, sex, and age are determinants of red blood cell storage and stress hemolysis: results of the REDS-III RBC-Omics study. Blood Adv. 2017;1(15):1132-1141.

34. Endres-Dighe SM, Guo Y, Kanias T, et al. Blood, sweat, and tears: Red Blood CellOmics study objectives, design, and recruitment activities. Transfusion. 2019;59(1):4656.

35. Lanteri MC, Kanias T, Keating S, et al. Intradonor reproducibility and changes in hemolytic variables during red blood cell storage: results of recall phase of the REDSIII RBC-Omics study. Transfusion. 2019; 59(1):79-88.

36. D'Alessandro A, Culp-Hill R, Reisz JA, et al. Heterogeneity of blood processing and storage additives in different centers impacts stored red blood cell metabolism as much as storage time: lessons from REDS III Omics. Transfusion. 2019;59(1):89-100.

37. Kanias T, Lanteri MC, Page GP, et al. Ethnicity, sex, and age are determinants of red blood cell storage and stress hemolysis: results of the REDS-III RBC-Omics study. Blood Adv. 2017;1(15):1132-1141.

38. Stone M, Keating SM, Kanias $\mathrm{T}$, et al. Piloting and implementation of quality assessment and quality control procedures in RBC-Omics: a large multi-center study of red blood cell hemolysis during storage. Transfusion. 2019;59(1):57-66.

39. Reisz JA, Barrett AS, Nemkov T, Hansen $\mathrm{KC}$, D'Alessandro A. When nature's robots go rogue: exploring protein homeostasis dysfunction and the implications for understanding human aging disease pathologies. Expert Rev Proteomics. 2018;15(4):293-309.

40. Karafin MS, Fu X, D'Alessandro A, et al. The clinical impact of glucose-6-phosphate dehydrogenase deficiency in patients with sickle cell disease. Curr Opin Hematol. 2018; 25(6):494-499.

41. Guo Y, Busch MP, Seielstad M, et al. Development and evaluation of a transfusion medicine genome wide genotyping array. Transfusion. 2019;59(1):101-111.

42. LaRue N, Kahn M, Murray M, et al. Comparison of quantitative and qualitative tests for glucose-6-phosphate dehydrogenase deficiency. Am J Trop Med Hyg. 2014; 91(4):854-861.

43. Arese P, Gallo V, Pantaleo A, Turrini F. Life and death of glucose-6-phosphate dehydrogenase (G6PD) deficient erythrocytes - role of redox stress and band 3 modifications. Transfus Med Hemother. 2012;39(5):328334.

44. Maurya PK, Kumar P, Chandra P. Age- 
dependent detection of erythrocytes glucose-6-phosphate dehydrogenase and its correlation with oxidative stress. Arch Physiol Biochem. 2016;122(2):61-66.

45. Kaestner L, Minetti G. The potential of erythrocytes as cellular aging models. Cell Death Differ. 2017;24(9):1475-1477.

46. Spencer BR, Bialkowski W, Creel DV, et al. Elevated risk for iron depletion in highschool age blood donors. Transfusion. 2019; 59(5):1706-1716.
47. Kanias T, Stone M, Page GP, et al. Frequent blood donations alter susceptibility of red blood cells to storage- and stress-induced hemolysis. Transfusion. 2019; 59(1):67-78.

48. Pietras EM, Mirantes-Barbeito C, Fong S, et al. Chronic interleukin-1 exposure drives haematopoietic stem cells towards precocious myeloid differentiation at the expense of self-renewal. Nat Cell Biol. 2016; 18(6):607-618.

49. DeSimone RA, Hayden JA, Mazur CA, et al. Red blood cells donated by smokers: A pilot investigation of recipient transfusion outcomes. Transfusion. 2019;59(8):2537 2543.

50. Nemkov T, Reisz JA, Xia Y, Zimring JC, D'Alessandro A. Red blood cells as an organ? How deep omics characterization of the most abundant cell in the human body highlights other systemic metabolic functions beyond oxygen transport. Expert Rev Proteomics. 2018;15(11):855-864. 